Rafał Hryszko

Uniwersytet Jagielloński

Instytut Historii

https://doi.org/10.18778/8220-478-0.07

\title{
LE RADICI MEDIEVALI DELLA CONFETTERIA ITALIANA
}

\begin{abstract}
La genesi dell'industria dolciaria è associata alla medicina, e in particolare alla farmaceutica, che ne è stata individuata. I prodotti a base di miele o di zucchero erano considerati al pari di medicine. Nel tardo medioevo i medicinali dolci cominciassero ad essere trattati come confetti. Quindi una parte importante della farmacia era basata sull'artificium zuchari, ovvero sulle regole per la preparazione di confetti, frutta candita, conserve e marzapane. Nel XV secolo i dolci costituivano una prova della posizione sociale e dell'importanza degli organizzatori e diventavano uno strumento di propaganda. Dopo la lunga lotta per il trono napoletano (1421-1442), il sovrano della Corona d'Aragona, Alfonso il Magnanimo (1416-1442-1458), portò in Italia elementi della cultura conviviale della di lui terra natale, compreso l'abitudine di un elegante e solenne rinfresco, chiamato colazione. Nella seconda metà del 400, la consumazione dei dolci era stata arricchita da elementi tipici italiani: dallo zucchero si potevano realizzare diverse cose di zuccaro, cioè figure, oggetti o composizioni.
\end{abstract}

Keywords: candy, sugar, speziare, colazione, Middle Ages, Renaissance

Streszczenie: Geneza cukiernictwa związana jest z medycyną, a zwłaszcza z wyodrębnioną z niej farmacją. Niektóre wyroby zawierające miód, a od średniowiecza także cukier, były uważane za lekarstwa. W późnym średniowieczu słodkie medykamenty zaczęto postrzegać jako słodycze. W efekcie istotna część ówczesnej farmacji zajmowała się tzw. artificium zuchari, czyli przygotowywaniem słodyczy, owoców kandyzowanych, konfitur i marcepanów. W XV wieku słodycze były dowodem pozycji i znaczenia organizatorów uczt i stały się narzędziem propagandy. Po długiej walce o tron neapolitański (1421-1442) władca Korony Aragonii, Alfons V Wspaniałomyślny (1416-1442-1458) przeszczepił do Italii elementy kultury biesiadnej wyniesione ze swojej ojczyzny, w tym zwyczaj uroczystej przekąski, zwanej z włoska colazione. $\mathrm{W}$ drugiej połowie XV wieku konsumpcję słodyczy wzbogacono o typowo włoskie elementy. $\mathrm{Z}$ cukru można było wytwarzać rozmaite cose di zuccaro, czyli postacie, przedmioty czy kompozycje cukrowe.

Słowa kluczowe: słodycze, cukier, średniowiecze, renesans 
Nei tempi moderni la produzione di vari tipi di dolci è appannaggio esclusivo dei pasticceri, che diventano dei veri maestri dell'arte dolciaria, come dimostrano sia le innumerevoli varietà di dolciumi, sia soprattutto la loro capacità di plasmare composizioni di zucchero e di altri ingredienti. Vale la pena di ricordare, tuttavia, che le loro abilità hanno avuto una evoluzione estremamente lunga, oltre che anche un po' complicata. Inoltre, quanto oggi l'industria dolciaria moderna può fare, non è il risultato dei progressi nel campo autonomo del settore industriale: la sua genesi è associata, invece, alla medicina $\mathrm{e}$ - in particolare - alla farmaceutica.

Quale ruolo ebbero i farmacisti italiani del primo Rinascimento nel processo di trasferimento delle regole per la produzione di dolciumi dalla sfera della farmacia al settore dolciario? Come si manifestò questo fenomeno? Perché le tradizioni della farmacia italiana del XIV e del XV secolo diventarono la base della moderna produzione dolciaria? - queste sono le principali domande che guideranno le nostre considerazioni.

Per affrontare i problemi in tal modo formulati, in primo luogo è necessario spiegare la specificità della produzione dolciaria medievale. Nella lingua polacca questo termine - conformemente alla definizione dell'Enciclopedia PWN - "significa l'arte di realizzare prodotti alimentari dolci: caramelle, cioccolato, pasticceria a lunga conservazione (ad es. pasticcini, biscotti) e quella a breve conservazione (ad es. paste e torte)" ${ }^{\prime}$. Se in Polonia, in linea di massima, viene usato un solo concetto generalizzante, nelle lingue romanze, invece, incluso l'italiano, questa sfera di produzione alimentare è definita con due termini separati che restringono e precisano l'ambito concettuale: il primo - la pasticceria - è più vicino alla sfera dei prodotti da forno e comprende le paste $^{2}$; il secondo ambito, invece - la confetteria - si riferisce direttamente alla produzione di dolciumi: caramelle, confetti, (nonché marmellate, masse di frutta, torroni ecc.) a base di miele, ma soprattutto a base di zucchero ${ }^{3}$.

Come possiamo vedere, la definizione italiana indica chiaramente entrambi i tipi specifici di prodotti e la loro stretta relazione con la materia prima di base che è lo zucchero. Il prodotto finale a questo punto sono i dolci (caramelle), che sono definiti come piccole caramelle formate da un nucleo centrale, come

1 Lemma: cukiernictwo, [in:] Encyklopedia PWN: https://encyklopedia.pwn.pl/ haslo/3888384/cukiernictwo.html [04/12/2019].

2 Lemma:pasticceria,[in:] Vocabolario Treccani:https://www.treccani.it/vocabolario/ pasticceria $[04 / 12 / 2019]$.

3 Lemma:confetteria, [in:] Vocabolario Treccani:https://www.treccani.it/vocabolario/ confetteria [04/12/2019]. 
mandorle, pistacchi, nocciole e altro, ricoperte da strati di zucchero più o meno denso, a volte colorato 4 .

Quindi, a quando risale la storia delle caramelle? Rispondere a questa domanda è una questione piuttosto complessa. Ciò è dovuto al fatto che i dolci di un lontano passato avevano un ruolo piuttosto diverso rispetto ai tempi moderni. Le loro origini, che risalgono alla preistoria e alla successiva era dell'antichità, erano associate all'emergere e al miglioramento dei metodi di conservazione degli alimenti, come l'essiccazione di frutta e di verdura o la loro conservazione in condizioni specifiche. Con il tempo, come conservanti cominciarono ad essere utilizzati il vino, l'aceto e soprattutto il miele. Quest'ultimo veniva usato come conservante per la frutta (tra cui mele, pere, melograni e cotogne), grazie ad esso si ottenevano masse dolci, conserve e frutta candita ${ }^{5}$.

Questi prodotti vennero riconosciuti dalle antiche autorità mediche (Ippocrate, Galeno) e considerati da loro come mezzi terapeutici. Questo significa che i prodotti dolci a base di miele (e dal Medioevo anche di zucchero) si trovarono associati per secoli prima alla medicina e poi alla farmaceutica, per cui essi venivano considerati e usati come prescrizioni farmaceutiche ${ }^{6}$.

Già nell'antichità, il consumo di tutti i prodotti fu strettamente soggetto alla cosiddetta teoria umorale, che nella sfera nutrizionale, che ci interessa, ad ogni sostanza alimentare attribuiva determinate proprietà. I prodotti contenenti miele (e zucchero) erano considerati secchi e caldi, quindi il loro consumo era raccomandato in inverno o a persone con il cosiddetto temperamento freddo, cioè agli anziani. Inoltre, le sostanze dolci vennero ampiamente utilizzate nei disturbi gastrici o nella loro prevenzione. Ciò era dovuto alla convinzione che marmellata, composte di frutta, torrone o marzapane facilitassero la digestione. Pertanto, il consumo di prodotti dolci era raccomandato prima e dopo i pasti. Allo stesso tempo, la combinazione di miele con varie spezie, e nei secoli

4 Lemma:confetto2,[in:] Vocabolario Treccani: https://www.treccani.it/vocabolario/ confetto2 [04/12/2019].

5 J. André (1961), L'alimentation et la cuisine à Rome, Paris, C. Klincksieck, pp. 209-212; L. Plouvier (1988), "La confiserie européenne au Moyen Âge”, Médium Aevum quotidianum, 13, p. 28; L. Plouvier (1992), "Le «letuaire», une confiture du bas Moyen Âge", [in:] Du manuscrit à la table, essais sur la cuisine au Moyen Age et répertoire des manuscrits médiévaux contenant des recettes culinaires, C. Lambert (a c. di), Montréal, Champion-Slatkine, Le Presses de l’Université de Montréal, pp. 243-245; M. Hyman (1992), "Les «menues choses qui ne sont de nécessité»: les confitures et la table", [in:] Du manuscrit à la table, essais sur la cuisine au Moyen Age et répertoire des manuscrits médiévaux contenant des recettes culinaires, C. Lambert (a c. di), Montréal, Champion-Slatkine, Les Presses de l'Université de Montréal, p. 275.

6 L. Plouvier (1988), op. cit., pp. 28-29; L. Plouvier (1992), op. cit., pp. 246-248. 
successivi anche con lo zucchero, rendeva il loro gusto più delicato e il loro consumo più fruibile ${ }^{7}$.

Nell'alto Medioevo, le ricette di medicine dolci iniziarono ad essere incluse in raccolte speciali di ricette di medicinali indicati per vari disturbi. Le più antiche nacquero nel mondo musulmano nel IX secolo e in arabo erano definite con il termine grabbadin, che significa una raccolta di farmaci composti (lat. composita).

Nel periodo tra l'XI e il XII secolo, la conoscenza di queste opere iniziò a diffondersi nell'Europa occidentale di cultura latina grazie all'attività dei due centri di traduzione: a Toledo, nella Penisola Iberica, e a Salerno, in quella appenninica. Il più celebre traduttore appartenente alla zona di Toledo fu Gerardo di Cremona, mentre Costantino Africano ${ }^{9}$ apparteneva alla seconda. Nel centro salernitano nacquero le raccolte separate di medicinali composti, denominati in latino antidotarii (Antidotarium magnum, Antidotarium Nicolai) ${ }^{10}$. In una di tali raccolte, un autore sconosciuto, sedicente scienziato musulmano

7 Kronika medycyny (1994), Warszawa, Kronika, pp. 54-55; R. Kuhne Brabant (1997), "Le sucre et le doux dans l'alimentation d’al-Andalus", Médiévales, 33, automne, p. 60; E. Savage-Smith (2005), Medycyna, [in:] Historia nauki arabskiej, Roshdi Rashed (a c. di), t. 3: Technika, alchemia, nauki przyrodnicze i medycyna, Warszawa, Wydawnictwo Akademickie Dialog, p. 171; Z. Gajda (2011), Do historii medycyny wprowadzenie, Kraków, Wydawnictwo WAM, pp. 106-108.

8 L. Plouvier (1988), op. cit., pp. 35-36; L. Plouvier (1999), "L'introduction du sucre en pharmacie”, Revue d'histoire de la pharmacie, 87 (332), p. 200; L. Plouvier (1992), op.cit., pp. 251-252; M. Ouerfelli (2008), Lesucre. Production, commercialisation et usage dans Méditerranée médiévale, Leiden-Boston, Brill, p. 512.

9 J.-P. Bénézet (1999), Pharmacie et médicament en Méditéranée occidentale (XIIIXVI siècles), Paris, Honoré Champion, pp.96-109; L. Cifuentes i Comamala (2006), La ciència en català a l'edat mitjana i el renaixement, Barcelona, Publicacions i edicions de la Universitat de Barcelona, p. 113; H. C. Silberman (1994), "Un électuaire d'Avicenne ou de la difficulté d'identifier les constituants de médicaments antiques", Revue d'histoire de la pharmacie, 82 (301), pp. 132-147; L. Plouvier (1999), op. cit., pp. 206-207; L. Plouvier (1992), op. cit., pp. 254-255; M. Ouerfelli, op. cit., pp. 523-525; D. Jacquart (2005), „Wpływ medycyny arabskiej na średniowieczny Zachód”, [in:] Historia nauki arabskiej, Roshdi Rashed (a c. di), t. 3: Technika, alchemia, nauki przyrodnicze $i$ medycyna, Warszawa, Wydawnictwo Akademickie Dialog, pp. 224-228.

10 P. Dorveaux (1896), L'Antidotaire Nicolas. Deux traductions françaises de l'Antidotarium Nicolai, l'une de XIV siècle, suivie de quelques recettes de la même époque et d'un glossaire, l'autre de XV siècle, incomplète, Paris, H. Welter, X, pp. 15-16, 23-24, 26-29; L. Plouvier (1999), op. cit., pp. 206-208; M. Ausécache (2007), "Manuscrits d'antidotaires médiévaux: quelques exemples du fonds latin de la Bibliothèque nationale de France", Médiévales, 52, printemps, pp. 55-62, 63-66; J.-P. Bénézet, op. cit., pp. 109-111; M. Ouerfelli, op. cit., pp. 523-524, 527. 
dal nome di Yuhanna ibn Masawaih (in lat. Johannes Mesues Damascenus, ca. 777-857), per la prima volta introdusse un capitolo a parte, con le ricette di medicinali prodotti a base di miele e di zucchero. Seguendo Lilianne Plouvier, le ricette di medicine dolci che vi erano raccolte possono essere divise in quattro gruppi: il primo è costituito da vari elettuaria, ovvero marmellate (ad esempio di mele, prugne, pesche, pere, mele cotogne), il secondo invece da frutta candita, preparata principalmente con lo zucchero: mele, mele cotogne, pèsche, pere e scorza di limone. Il terzo include i farmaci in forma di liquido molto denso; il quarto, sciroppi ed estratti di succo di frutta ${ }^{11}$.

Le opere di cui sopra erano destinate ai farmacisti dell'epoca. Anche in questo caso, l'ambito della loro attività professionale differiva significativamente da ciò che possiamo immaginare ai nostri tempi. Negli stati italiani medievali i farmacisti erano chiamati speziari. Il nome è associato al termine spezie, che è

denominazione generica, per lo più al plurale e con valore collettivo, di diverse sostanze aromatiche di origine vegetale (pepe, zenzero, chiodi di garofano, cannella, noce moscata, ecc.), generalmente di provenienza esotica, usate, oltre che per aromatizzare e insaporire cibi e bevande, anche, spec. in passato, in medicina e in farmacia ${ }^{12}$.

In questa prospettiva speziare (oggi: speziale) era un venditore di sostanze menzionate ${ }^{13}$. Oltre alle capacità concrete e una bottega ben attrezzata, la condizione indispensabile per svolgere l'attività del genere era l'appartenenza a un'adeguata arte (per esempio a Firenze l'arte dei medici e speziari), volta a regolare rigorosamente l'ambito delle prerogative dei suoi membri in base alle norme legali applicate all'epoca. Queste includevano sia statuti di corporazione, ordinanze comunali e legislazione statale ${ }^{14}$.

11 L. Plouvier (1988), op. cit., pp. 42-45; L. Plouvier (1992), op. cit., pp. 255-256; L. Plouvier (1999), op. cit., pp. 209-212; L. Plouvier (2006), "Le rôle d’al-Andalus dans la transmission des connaissances de l'Orient vers l'Occident: l'exemple e la confiserie”, Horizons maghrébins, 55, pp. 42-43; M. Ouerfelli, op. cit., p. 530.

12 Lemma:spezie, in: Vocabolario Treccani: https://www.treccani.it/vocabolario/ spezie $[04 / 12 / 2019]$.

13 Lemma:speziale2, in: Vocabolario Treccani: https://www.treccani.it/vocabolario/ speziale; www.treccani.it/vocabolario/speziale2 [04/12/2019].

14 A. Giuffrida (1976), La bottega dello speziale nelle città siciliane del'400, [in:] Atti del Colloquio Internazionale di Archeologia Medievale, Palermo-Erice, 20-22 settembre 1974, Palermo, Istituto di storia medievale, Università di Palermo, pp. 465-504; R. Ciasca (1927/1977), L'arte dei medici e speziali nella storia e nel commercio fiorentino dal secolo XII al XV, Firenze, Leo S. Olschki Editore. 
In pratica, l'ambito dell'attività degli speziari era molto più ampio: oltre alla distribuzione delle spezie menzionate sopra, nelle botteghe degli speziari era possibile acquistare sia medicinali semplici (in latino simplicia), sia composti (in latino compositi). Questi ultimi venivano preparati secondo le indicazioni contenute nelle ricette, tenendo conto delle norme di qualità imposte dalla corporazione professionale. Inoltre, vi si realizzavano unguenti, profumi e candele e a quell'epoca vi si potevano acquistare i fogli di carta e inchiostro di vari colori. La produzione di dolci fu unaltra importante sfera dell'attività speziaria del XIV e specialmente nel XV secolo ${ }^{15}$.

In quest' ultimo caso, è difficile dire in modo inequivocabile quando alcuni prodotti realizzati dagli speziari abbiano cominciato ad essere considerati dolci. Certamente non è privo di significato ${ }^{16}$ il fatto che i commercianti italiani, principalmente veneziani e genovesi, importassero miele e soprattutto zucchero dal Medio Oriente e da Cipro, Granada (XV sec.), Madera e Isole Canarie.

Sembra che questo processo già avvenisse almeno nel XIII secolo e che l'aumento del consumo di zucchero possa essere direttamente correlato alla crescente domanda di prodotti su di esso basati. Non senza importanza era il fatto che allora sempre più spesso si prestava l'attenzione al sapore dello zucchero. Inoltre, una predilezione speciale per lo zucchero e i prodotti dolci faceva sì che le raccomandazioni dietetiche di allora, risultanti dai principi della teoria umorale, fossero spesso respinte. Quindi non sorprende che il medico di Milano, Maino de Marinieri (1290/1295-1365/1368) noto anche come Maginus Mediolanensis, nel suo lavoro intitolato Liber regiminis sanitatis, scritto a Parigi negli anni 1331-1334, presentando il concetto di sanitas, e le indicazioni dettagliate da utilizzare per rimanere in salute, prestò una particolare attenzione al cambiamento delle regole di consumo dei dolci ${ }^{17}$.

Nel capitolo 26 (nella terza parte del suo lavoro), dal titolo De confectionibus et earum usu, Maino de Maineri dedica ampio spazio alla descrizione particolareggiata dei dolci. Già nella prima frase del capitolo mette in rilievo il fenomeno comune a quell'epoca, relativo alle persone sane che ricorrevano al consumo di vari dolci, indipendentemente dai pasti assunti o dalla stagione.

15 J.-P. Bénézet, op. cit., pp. 185-187; D. Santoro (2006), "Lo speciale siciliano tra continuità e innovazione: capitoli e costituzioni dal XIV al XVI secolo”, Mediterranea. Ricerche storiche, 3, dicembre, p. 469.

16 L. Plouvier (1999), op. cit., p. 201; R. Kuhne-Brabant (1997), op. cit., p. 60; M. Ouerfelli, op. cit., pp. 541-542.

17 M. Palumbo, Maineri, Maino (Manio), [in:] Dizionario Biografico degli Italiani: https://www.treccani.it/enciclopedia/maino-maineri_(Dizionario_Biografico) [10/11/2019]. 
Questo fatto divenne per lui l'impulso a riflettere sul tema dei dolci (Quia homines sani communiter utuntur confectionibus et ante cibum et post, et stomacho pleno et vacuo, et tempore calido et frigido, bonum mihi videtur de confectionibus hic facere unum capitulum speciale). Secondo il medico italiano i medicinali dolci venivano consumati non per necessità terapeutiche ma per pura golosità (Scienum igitur quod homines communiter utunur in sanitate forum confectionibus magis ad voluntatem quam propter necessitatem-sottolineato da R.H. ${ }^{18}$.

Certamente le motivazioni descritte da Maino Maineri contribuirono al consolidamento e all'aumento dell'importanza di prodotti, il cui ingrediente principale era lo zucchero. D'altra parte, senza dubbio illustrano il cambiamento dell'atteggiamento nei confronti dei medicinali dolci, che andò sviluppandosi nel tardo medioevo, i quali già allora cominciarono ad essere trattati come confetti. Come sottolinea Claudio Benporat, il termine confetti può essere inteso in due modi: nel senso più stretto del termine, si trattava di un prodotto con un nucleo duro coperto di zucchero; il nucleo duro dei confetti era costituito da vari tipi di cereali o semi (ad es. coriandolo, anice), spezie intere o in frammenti - cannella, cardamomo, noce moscata (macis), zenzero, uva passa, cubebe nonché mandorle e noci ${ }^{19}$.

Nel senso più ampio del termine, confetti o confezioni indicavano come prodotti a base di frutta, tanto frutta candita (canditi) quanto gelatine ${ }^{20}$. Le fonti prese in esame indicano che nei singoli stati italiani venivano consumati confetti definiti come traggea (triggea) ${ }^{21}$. Le fonti menzionate presentano i nomi propri dei dolci, tra cui coriandri $i^{22}$, anisi ${ }^{23}$, canellini $i^{24}$, oppure grissini di zucchero,

18 Regimen sanitatis Magini mediolanensis cum nonnulis insuper Auicenne ac plerumq[ue] aliorum auctoru[m] margine cartharum insertis; Insuper opusculum de fleubothomia editu[m] a Reginaldo de Villa noua, (1506), per Gaspardum Philips, expensis Iohannis Petit, Parisium, XLVIII.

19 C. Benporat (2005), "Note per la storia dei confetti", Appunti di gastronomia, 47, pp. 71-72.

20 E. Carnevale Schianca (2011), La cucina medievale. Lessico, storia, preparazioni, Firenze, Leo S. Olschki Editore, pp. 178-179.

21 C. Benporat (2001), Feste e banchetti. Convivialità italiana fra Tree Quattrocento, Firenze, Leo S. Olschki Editore, pp. 123, 126, 135-136, 139, 145, 149; E. Carnevale Schianca, op. cit., p. 678.

22 Q. de Augustis (1492), Lumen apothecariorum, Taurini, XXXVv; C. Benporat (2001), Feste e banchetti..., pp. 160, 175, 235.

23 Q. de Augustis, op. cit., XXXVv-XXXVIr; E. Carnevale Schianca, op. cit., pp. 38-39; C. Benporat (2001), Feste e banchetti..., pp. 160, 175, 235.

24 Q. de Augustis, op. cit., XXXVv; C. Benporat (2001), Feste e banchetti..., p. 160; E. Carnevale Schianca, op. cit., p. 110. 
chiamati manus Christi (Menechristi indorati in tazze d'oro avanti pasto ${ }^{25}$ spesso coperti con scaglie d'oro ${ }^{26}$.

L'élite italiana, a cui piacevano i dolci, non disprezzava il consumo dei chicchi di frutta candita ${ }^{27}$. Per preparare questo tipo di pasticceria venivano usati sia i frutti dalla consistenza dura, sia quelli dalla consistenza molle. Il primo gruppo comprendeva mandorle confette ${ }^{28}$, mandorle col zuccharo o mele ${ }^{29}$, pinoli $^{30}$ e nocciole ${ }^{31}$.

Un gruppo a parte dei canditi veniva costituito da cedri (le ricette in Anonimo Padovano, manoscritto dell'abbazia di Morimondo ${ }^{32}$ ), mele (Ambrogio de Oderico $^{33}$, Anonimo veneziano ${ }^{34}$ ) arance $^{35}$ intere oppure la loro buccia, nonché gli ortaggi come ad esempio le zucche, di cui si facevano confetture (la ricetta in Anonimo lucano) ${ }^{36}$.

Anche altri frutti venivano sottoposti al processo di canditura: nelle descrizioni delle feste del tardo Medioevo troviamo: pere (pere confecte, pere confecte con çuccaro, pera guaste cum zucaro in paiatelli, pera muscatela in confetere $^{37}$ ), limoncioni confecte ${ }^{38}$, limoni sciroppati inargentati ${ }^{39}$, melagrane

25 Q. de Augustis, op. cit., XXXVIr-XXXVIv; C. Benporat (2001), Feste e banchetti..., p. 173.

26 E. Carnevale Schianca, op. cit., pp. 381-382.

27 E. Carnevale Schianca, op. cit., p. 179.

28 Q. de Augustis, op. cit., XXXVv-XXXVIr; C. Benporat (2001), Feste ebanchetti..., p. 154.

29 C. Benporat (2001), Feste e banchetti..., p. 136.

30 Ibidem, pp. 160, 153-154, 175, 255.

31 E. Faccioli (1966), Arte della cucina, vol. I, Milano, Edizioni il Polifilio, p. 102; E. Carnevale Schinaca, op. cit., pp. 380, 438.

32 E. Carnevale Schianca, op. cit., pp. 147-148, XIV.

33 L. Balletto (1986), Medici e farmaci, scongiuri ed incantesimi, dieta e gastronomia nel medioevo genovese, Genova, Università di Genova, p. 244; E. Carnevale Schianca, op. cit., p. 386.

34 E. Faccioli, op. cit., p. 104, n. CXXXII; E. Carnevale Schianca, op. cit., p. 389.

35 E. Faccioli, op. cit., pp. 103-104, num. CXXX; Maestro Martino (1996), Ms. Bühler 19, New York, Pierpont Mogran Library, [in:] C. Benporat, Cucina italiana del Quatrocento, Firenze, Leo S. Olschki Editore, p. 282; E. Carnevale Schianca, op. cit., p. 548.

36 E. Faccioli, op. cit., p. 103, num. CXXIX; L. Mancusi Sorrentino (1993), "Apparecchi diversi da mangiare et rimedii", Appunti di gastronomia, 11, pp. 40-42; C. Benporat (2001), Feste e banchetti..., p. 154.

37 C. Benporat (2001), Feste e banchetti..., pp. 123, 126-127, 282, 284, 289.

38 Ibidem, p. 262.

39 Ibidem, p. 174. 
(melagrane inzucarate ${ }^{40}$ ), olive (ulive confette) ${ }^{41}$, finocchio dorato in zucchero (finochii coperti de zucharo et dorati in altre confectiere, finoghio inzucarato et indorato $)^{42}$, amarene (cerase in confetere $)^{43}$.

A volte dal succo e dalla polpa di frutta schiacciata, mescolata con spezie e miele o zucchero, venivano preparati prodotti con la consistenza della gelatina: questo gruppo comprendeva confetture di mele cotogne (cotognata), gelatina di arance ${ }^{44}$ e di pesche ${ }^{45}$, che non si servivano in appositi recipienti, ma venivano tagliate a pezzi (cubi o rombi). Questi ultimi erano avvolti in zucchero mischiato a spezie in polvere ${ }^{46}$.

Il marzapane appartiene all'ultimo gruppo di dolci popolari italiani. Le menzioni frequenti del loro consumo durante le feste - ad esempio nelle fonti raccolte da C. Benporat si verificano circa 17 rinvenimenti-possono confermare l'eccezionale predilezione per questo tipo di dolci ${ }^{47}$. Il marzapane aveva diverse forme. Si consumava sia in pezzi piccoli e sottili, sia grandi e dorati ${ }^{48}$. Il Maestro Martino, autore di uno dei compendi culinari italiani, indica che il marzapane venne usato per decorare i piatti (marzipani dece cum arme del principe $)^{49}$. Una pasta di lavuri de zuccaro, che era una specie di massa di marzapane, può essere considerata un prodotto simile allo stesso marzapane (Anonimo lucano) ${ }^{50}$.

Una breve descrizione di prodotti degli speziari italiani, di cui sopra, certamente non esaurisce il quadro generale della questione. Un altro passo

40 Ibidem, pp. 167, 173, 189.

41 Ibidem, p. 251.

42 M. Maestro, op. cit., pp. 284, 287; 289; C. Benporat (2001), Feste e banchetti..., p. 160.

43 Maestro Martino, op. cit., pp. 283, 286.

44 E. Faccioli, op. cit., p. 104, num. CXXXI; E. Carnevale Schianca, op. cit., p. 548; G. Palmero (1998), Entre culture thérapeutique et culture matérielle: les domaines de savoir d'un anonyme gênois à la fin du Moyen-Âge. Le manuscrit inédit „Medicinalia quam plurima", vol. II, thèse de doctorat sous la direction de Henri Bresc, pp. 199-200, \$ 795 [fol. 170r]; 266, \$ 1233 [fol. 227 v].

45 E. Carnevale Schianca, op. cit., pp. 179, 190-191.

46 E. Faccioli, op. cit., pp. 104-105, num. CXXXIII, C. Benporat (2001), Feste e banchetti..., p. 256; E. Carnevale Schianca, op. cit., p. 191; L. Mancusi Sorrentino, op. cit., pp. $35,89$.

47 C. Benporat (2001), Feste e banchetti..., pp. 142, 154, 170-171, 175, 193, 215, 235, 250, 252, 275; Maestro Martino, op. cit., pp. 284, 287, 289; E. Carnevale Schianca, op. cit., p. 383; B. Maggi (2001), "Il marzapane", Appunti di gastronomia, 34, pp. 73-84; S. Leone (2009), "Alcuni appunti sul torrone”, Appunti di gastronomia, 58, pp. 83-86; F. Sorrentino (2001), "Le nozze Trivulzio-d'Avalos e la pignolata napoletana”, Appunti di gastronomia, 44, pp. 57-62.

48 C. Benporat (2001), Feste e banchetti..., pp. 159, 193, 239.

49 Maestro Martino, op. cit., p. 291; Q. de Augustis, op. cit., XXXVIv.

50 L. Mancusi Sorrentino, op. cit., pp. 84-85; E. Carnevale Schianca, op. cit., p. 724. 
nella ricerca delle caratteristiche specifiche della pasticceria italiana può essere quello di confrontare la gamma di dolci italiani, ad esempio con prodotti dolci degli especiers catalani. Ciò consentirà di cogliere non solo alcune differenze chiaramente visibili, ma darà anche la possibilità di indicare le specificità dellassortimento di prodotti degli speziari italiani.

A prima vista, i prodotti di entrambe le aree mostrano notevoli analogie sia per quanto riguarda i tipi che le regole della loro preparazione. Tuttavia, un'attenta analisi del contenuto delle ricette italiane e catalane conservate finora, mostra che una caratteristica visibile fu il predominio dello zucchero nelle regioni dell'Italia settentrionale e centrale nei secoli XIV e XV. A differenza della Corona d'Aragona, la percentuale di miele negli ingredienti per la preparazione dei vari di dolci italiani è praticamente trascurabile. Ciò si deve al fatto che in Italia i dolci in forma di caramella avevano un ruolo molto più importante rispetto alle aree di lingua catalana - dove in luogo della produzione se ne verifica praticamente solo l'importazione, dal Medio Oriente. Inoltre, in Italia, la gamma di tali dolci era decisamente più ampia. Inoltre, i prodotti degli speziari italiani si caratterizzavano per la grande raffinatezza che riguarda la qualità, le forme e le decorazioni - come ad esempi l'utilizzo di scaglie d'oro ${ }^{51}$.

Un altro aspetto, la cui analisi ci consentirà di individuare le caratteristiche specifiche della produzione dolciaria italiana, sarà il contesto del consumo. In primo luogo, mangiare i dolci era condizionato dalle circostanze del calendario liturgico della Chiesa: i dolci venivano consumati sia durante le festività natalizie e pasquali, sia in occasione del Santo patrono della determinata città ${ }^{52}$. Altre occasioni erano legate all'assegnazione di alte cariche pubbliche o di importanti mansioni, inquantoché eventi di questo tipo erano sempre accompagnati da feste sontuose ${ }^{53}$. Si servivano dolci anche nel contesto di ricevimenti ufficiali di natura pubblica, come visite di teste coronate - imperatori, re o principi

51 R. Hryszko (2013), Media aeva dulcia. Analiza produkcji i konsumpcji w Koronie Aragonii w XIV i XV w., Kraków, Towarzystwo Wydawnicze "Historia Iagellonica", pp. 249-250.

52 A. Vigna (1879). Codice diplomatico delle colonie tauro-liguri durante la signoria dell'Ufficio di San Giorgio (MCCCCLIII-MCCCCLXXV), t. II, p. II (anni 1473-1475). Atti della Società Ligure di Storia Patria, vol. VII, p. II, fasc. I-II, p. 617, num. 231; R. Hryszko (2012), Na kaffeńskim stole-wyimek z problematyki żywieniowej czarnomorskich kolonii Genui w późnym średniowieczu, [in:] Ł. Gędłek, T. Krzyżowski, M. Michalski (a c. di), Regiones Euxinum Spectantes. Stosunki kulturowe, etniczne i religijne na przestrzeni dziejów, Kraków, Uniwersytet Papieski Jana Pawła II, pp. 249, 252.

53 C. Benporat (2001), Feste e banchetti..., pp. 36, 142-143; C. Benporat (2005), "Note per la storia dei confetti”, Appunti di gastronomia, 47, p. 73; C. Benporat (2009), "Convivialità degli ultimi Visconti", Appunti di gastronomia, 58, pp. 5-16. 
- nonché alti funzionari della Chiesa o visite di delegazioni ufficiali ${ }^{54}$. Si offrivano dolci anche durante le cerimonie nuziali: in questo caso, l'abitudine di mangiare i dolci diventò un attributo indispensabile delle feste di nozze, riflesso nel detto: mangiare $i$ confetti, che significa la relazione attesa dei due persone $e^{55}$. Quindi, nell'Italia del Tre- e del Quattrocento non mancavano le occasioni per festeggiare e ogni celebrazione non poteva fare a meno del consumo di dolci.

Laumento della popolarità dei dolci contribuì a consolidare nuove forme di cultura del banchetto italiano, sotto forma di uno spuntino dolce - la colazione.

Le sue origini risalgono alle pratiche di digiuno frequenti nell'ambiente del monastero. Nei monasteri benedettini c'era una regola quaresimale di astenersi dal mangiare qualsiasi cibo prima che tramontasse il sole. Tuttavia, in condizioni climatiche difficili e con un intenso lavoro, la mancanza di assunzione del cibo diventò un problema molto serio. In questo contesto, in un periodo non meglio precisabile, ebbe origine l'usanza di consumare uno spuntino pomeridiano leggero, cioè la collatio (il cui termine trae origine dalla lettura di Collationes patrum di Giovanni Cassiano - ca 360-435), durante cui si mangiava pane con vino e acqua. Nel corso del tempo, l'abitudine di tale pasto veloce e leggero si diffuse al di fuori dellambiente ecclesiastico, con la sostituzione del pane con i dolci. Quando i dolci cominciarono a godere di una crescente popolarità, anche la colazione dolce venne organizzata durante tutto l'anno, indipendentemente dalle iniziali pratiche di digiuno ${ }^{56}$.

Allo stato attuale dei lavori di ricerca, determinare il momento in cui si verificò questo processo è estremamente difficile. Tuttavia, si può presumere che l'apparizione delle nuove forme di consumo di dolciumi fosse associata alla diffusione dello zucchero, avvenuta nel XIV secolo. La quantità dei documenti provenienti dal periodo a cavallo tra il XIV e il XV secolo indica che tale modo

54 L. A. Gandini (1899), Tavola, cucina e cantina della corte di Ferrara nel Quattrocento. Saggio storico, Modena, Società tipografica modenese, pp. 39-41; A. Cougnet (1923), L'evoluzione dell'arte dulciaria in Italia, [in:] G. Ciocca, Il pasticciere e confettiere moderno. Raccolta completa di ricette, Milano, Ulrico Hoepli, pp. LIII-LIV, LXIX; C. Comolli (1991), "Cucina e feste presso la corte ferrarese del'400”, Appunti di gastronomia, 6, pp. 27-29.

55 A. Cougnet, op. cit., pp. LXIII-LXIV.

56 C. Vela i Aulesa (1996), "La col-lació, un apat medieval poc conegut", [in:] La Mediterrània. àrea de convergència de sistemes alimentaris (segles V-XVIII). XIV Jornades d'Estudis Històrics Locals: realizades a Palma del 29 de novembre al 2 dicembre de 1995), Palma, Consejo Superior de Investigaciones Sientíficas, pp. 669-670; E. Carnevale Schianca, op. cit., p. 168; S. Claramunt (1988), "Dos aspectes de l'alimentació medieval: dels canonges a les "miserabiles personae", [in:] Alimentació i societat a la Catalunya medieval. Anuario de estudios medievales, Anex 20, Barcelona, Consejo Superior de Investigaciones Sientíficas, p. 170. 
di festeggiare veniva praticato negli ambienti dell'élite finanziaria e quella dei potenti e dei sovrani di quel tempo. Quindi la colazione dolce conobbe il suo rinascimento nel Quattrocento, quando acquistò sia un nuovo significato che un'ambientazione appropriata ${ }^{57}$. Nella letteratura sull'argomento, le menzioni riguardanti le colazioni solenni negli stati italiani risalgono soltanto agli anni '70 del XV secolo. Nonostante ciò, un'attenta analisi delle fonti ci consente di avanzare un'ipotesi abbastanza prudente secondo la quale la diffusione di uno degli elementi importanti della cultura del banchetto italiano dell'età del Rinascimento, ovvero una celebrazione ostentata di prodotti dolci, ebbe luogo a cavallo tra gli anni '30 e '40 del XV secolo e fu merito del sovrano della Corona d'Aragona, Alfonso il Magnanimo (1416-1442-1458). Quest'ultimo, dopo esser salito al potere nella sua patria nel 1416, dall'inizio degli anni '20 del XV secolo si impegnò nella lotta di successione per il trono napoletano, dopo il regno di Giovanna II (1414-1435). Il conflitto iniziò nel 1421 e continuò con delle pause fino al 1442, quando fu concluso con pieno successo per il sovrano aragonese, che pure significò il suo dominio sull'Italia meridionale ${ }^{58}$. Le fonti conservate indicano chiaramente che Alfonso aveva una predilezione particolare per i dolci. Ciò è dimostrato da dati e cifre e anche dal fatto che venivano ripetutamente inviati dolci nei luoghi in cui il sovrano della Corona d'Aragona e del Regno di Napoli si trovava ${ }^{59}$. Quindi, come si può presumere, questa predilezione del sovrano per i dolci, nonché le ragioni della propaganda, possono spiegare il fatto che il re proveniente dall'ambiente culturale catalano avesse dato un nuovo significato all'usanza della merenda dolce praticata nella Penisola Appenninica. Come testimoniano gli autori citati sopra, e prima di tutto Giovanni Pontano, proprio Alfonso il Magnanimo contribuì direttamente al trasferimento in Italia dell'usanza dell'ostentata celebrazione delle col-lació catalane $^{60}$. Ma quale carattere avevano queste celebrazioni organizzate nella Corona d'Aragona a cavallo tra il XIV e il XV secolo?

57 E. Carnevale Schianca, op. cit., p. 168.

58 R. Muscati, Alfonso V d'Aragona, re di Sicilia, re di Napoli, in: Dizionario Biografico degli Italiani: https://www.treccani.it/enciclopedia/alfonso-v-d-aragonare-di-sicilia-re-di-napoli_(Dizionario-Biografico) [10.11.2019]; Alfonso V, [in:] Diccionario Biográfico electrónico: http://dbe.rah.es/biografias/6367/alfonso-v [10.11.2019].

59 J. Guiral (1984), "Le sucre à Valence aux XV et XVI siècles", [in:] Manger et boire au Moyen Âge. Actes du Collque de Nice (15-17 octobre 1982), t. 1: Aliements et Societé, Nice, Les Belles Lettres, pp. 119-129; M. Ouerfelli, op. cit., p. 637.

60 G. G. Pontano [Ioannis Ioviani Pontani] (1518), Opera omnia soluta oratione composita, t. I, Venetiis, p. 14; C. Benporat (2001), Feste e banchetti..., pp. 93-99; C. Benporat (2001), "De conviventia, un trattato di etica conviviale di Giovanni Pontano", Appunti di gastronomia, 36, pp. 10-16; C. Benporat (2007), "La „collatione”. una nuova forma conviviale", Appunti di gastronomia, 52, pp. 45-58. 
In base alle informazioni che si trovano nelle fonti catalane si può costatare che le feste solenni con i dolci organizzate nei territori catalani vennero celebrate almeno dagli anni '70 del XV secolo. Le organizzavano non solo i sovrani della Corona d’Aragona, ma anche le autorità delle singole città, tra cui Barcellona, di solito in occasione delle visite reali e delle missioni diplomatiche straniere. Durante queste feste, venivano serviti solo il vino e i dolci, ma le cerimonie avevano il carattere particolarmente solenne, e qui la danza era un elemento importante ${ }^{61}$.

Di tale modo di celebrare il rinfresco con i dolci negli anni ' 40 e ' 50 del $\mathrm{XV}$ secolo, parlavano le relazioni dei rappresentanti delle autorità comunali di Barcellona, che - nell'ambito delle regolari missioni - accompagnavano il re durante la seconda spedizione in Italia e anche dopo l'occupazione di Napoli (quindi negli anni 1435-1458). Altro tipo di relazioni costituiscono quelle degli umanisti legati con la corte di Napoli tra cui: Antonio Beccadelli, detto il Panoramita (1394-1471), Bartolomeo Facio (ca. 1400-1457) e Giovanni Pontano (1429-1503) ${ }^{62}$. Alla luce delle informazioni provenienti dalle fonti, possiamo constatare in modo certo che Alfonso organizzò dei rinfreschi solenni con i dolci tanto negli anni '40 quanto anche negli anni '50 del XV secolo. Le occasioni non mancavano. Le colazioni solenni ebbero luogo durante i negoziati diplomatici con l'inviato del signore di Ferrara, Leonello d'Este (1407-1450) riguardanti il suo matrimonio con Eleonora, figlia minore di Alfonso V (alla fine Leonello d'Este sposò la figlia maggiore - Maria) ${ }^{63}$, durante il battesimo della nipote di Alfonso - Eleonora, figlia del figlio illegittimo del re Ferdinando, detto Ferrante, avvenuto il 2 agosto $1450^{64}$ e durante una visita dell'imperatore Federico III a Napoli nel $1452^{65}$. Invece Giovanni Pontano indicò chiaramente che proprio Alfonso decise del ruolo propagandistico della colatione con dolci, i cui valori visuali avrebbero dovuto avere il ruolo di primo piano ${ }^{66}$.

61 R. Hryszko (2013), op. cit., pp. 214-216, 218, 220-224; R. Hryszko, R. Sasor (2017), Średniowieczne słodycze katalońskie $w$ źródłach $i$ literaturze ( $z$ wyborem tekstów z XIV i XV wieku), Kraków, Towarzystwo Wydawnicze Historia Iagellonica, pp. 192-215.

62 R. Hryszko (2019), „The Sweet War, or How Alfons V of Aragon's Military Campaigns Affected the Eating Habits in Early to Mid-15th Century”, Perspektywy kultury, nr 26 (3/2019), pp. 146-149.

63 Mensajeros barceloneses en la corte de Nápoles de Alfonso V de Aragón (1963), Introducción y texto, cfr. J.M. Madurell Marimón, Barcelona, Consejo Superior de Investigaciones Sientíficas, p. 227, num. 169.

64 Ibidem, p. 311, num. 249.

65 A. Beccadelli (1990), Dels fets e dits del gran rey Alfonso, Barcelona, Editorial Barcino, pp. 256-259, 264-267; B. Faccio (1590), I fatti d'Alfonso d'Aragona primo re di Napoli, Vinegia, Apresso Giovanni et Gio. Paolo Giolitti de Ferrari, p. 418.

66 G. G. Pontano, op. cit., p. 14; C. Benporat (2001), De conviventia... pp. 10-16; C. Benporat (2007), op. cit., pp. 45-58; C. Benporat (2001), Feste e banchetti..., pp. 93-99. 
Le menzioni di cui sopra indicano chiaramente che il primo monarca dell'Italia del Sud dalla dinastia aragonese, dopo una lunga lotta per il potere a Napoli e la sua conquista, portò in Italia certi elementi della cultura conviviale della sua terra natale, tra cui un ruolo importante spettò non solo ai dolci ma anche almodo in cui venivano serviti e mangiatinel corso di un elegante e solenne rinfresco. Diede all'evento in sé un significato particolare, creò il modello di comportamento, imitato subito tanto dai sovrani italiani rinascimentali, quanto dagli aristocratici. Grazie ad Alfonso V il Magnanimo, la colazione divenne una parte indissolubile della cultura conviviale italiana, praticata spesso presso le corti dei principi nel primo Rinascimento.

Ad esempio, nel 1475 nel corso di una colazione durante la cerimonia nuziale di Costanzo Sforza e di Camilla d'Aragona, una grande impressione sui partecipanti fece il corteo di ottanta servitori che portavano nella sala da ballo numerosi panieri con vari tipi di dolci, e un altro, in cui si mostrò anche la presenza di un cammello $[\ldots]$

si ben contraffatto e con tanta arte, che pareva vivo, ed era grande e apria la bocca e distendeva il collo e coricavasi in terra come fanno li veri camelli, ed era carco di due grandissime ceste d'oro piene e colme di varie confezioni, e in mezzo del cammello era uno garzone etiopo negro che mettea amendue le mani mo' nell'una e mo' nell'altra in queste ceste, e sprageva e buttava detti confetti al popolo e per tutta la sala: che era bellissima cosa a vedere ${ }^{67}$.

I dolci non erano un semplice piatto servito per soddisfare i raffinati gusti dei convitati. Le quantità servite, la loro varietà, il modo particolarmente solenne con cui venivano serviti, erano una prova della posizione e dell'importanza degli organizzatori - rappresentanti delle più rinomate casate principesche, aristocratici o ecclesiastici. Spesso le composizioni dei dolci diventavano uno strumento di propaganda, dato che matrimoni e banchetti di nozze servivano anche per consolidare le alleanze politiche. A questi fini, nell'Italia quattrocentesca e forse anche prima, si utilizzavano le proprietà plastiche dello zucchero - già conosciute nella medicina islamica dal pieno Medioevo e in seguito adoperate anche dalla farmacia occidentale - per realizzare le opere la cui forma dipendeva strettamente dal contesto ideologico o situazionale.

Dallo zucchero sottoposto a trattamento termico si otteneva la pasta di zucchero chiamata fanid oppure panid, in latino penidium. Per preparare la pasta era indispensabile lo sciroppo d'acqua e di zucchero, che veniva riscaldato alla temperatura dai 121 gradi C. ai 143, e in seguito sottoposto ad evaporazione,

67 C. Benporat (2001), Feste e banchetti..., pp. 210, 215; A. Cougnet (1923), op. cit., p. LX; C. Benporat (2005), op. cit., p. 86. 
grazie alla quale acquisiva proprietà plastiche ${ }^{68}$. Quando il semiprodotto liquido veniva lasciato su una fredda piastra di marmo cospersa di amido, la massa poteva essere plasmata e alla fine irrigidiva, così da poterne ricavare diverse cose di zuccaro cioè figure, oggetti o composizioni ${ }^{69}$.

Dallo zucchero riscaldato e diluito venivano formati diversi oggetti, anche figure umane o allegoriche. Queste composizioni si riferivano agli eventi che venivano celebrati. Nel 1473 al banchetto organizzato dal cardinale Girolamo Riario, sui tavoli vennero messi i piatti per i dolci con modellini in zucchero di navi ${ }^{70}$. Dopo due anni, alle nozze di Costanzo Sforza con Camilla d'Aragona, venne presentata una collina portata dalle tre damigelle che personificavano la Grammatica, la Retorica el'Astrologia. Sulla sua cima si trovavano delle figurine di zucchero che rappresentavano Apollo con le muse danzanti, nonché il gruppo di poeti greci e latini con i libri di zucchero in mano. Dalla pasta di zucchero si potevano plasmare anche altre forme. Lo zucchero servì per preparare un carro trionfale tirato da buoi ${ }^{71}$. Nel 1493, le autorità di Venezia ordinarono agli speziari locali una composizione con numerose figure che rappresentassero il Papa, alcuni sovrani italiani o lo stemma di Venezia e di Milano ${ }^{72}$.

Un gruppo a parte era costituito dalle composizioni fatte per intero dalla pasta di zucchero, tra cui castelli di zucchero, o modelli che imitavano

68 L. Plouvier (1988), op. cit., p. 38; L. Plouvier (1999), op. cit., pp. 201-202; L. Plouvier (1992), op. cit., p. 254; L. Plouvier (2006), op. cit., pp. 36-37.

69 E. Savage-Smith (2005), op. cit., pp. 180-181; L. Plouvier (1988), op. cit., pp. 35-36; L. Plouvier (1992) op. cit., p. 252; L. Plouvier (2006) op. cit., pp. 34-37.

70 Notabilia temporum di Angelo de Tummulillis da Sant'Elia (1890), C. Corvisieri (a c. di), Roma, Tipografia Francesco Vigo, pp. 194-203; C. Benporat (1999), "Decori, castelli e statue di zuccharo sulla scena conviviale italiana", Appunti di gastronomia, 30, p. 55.

71 E in mezzo era il fonte Elicona con uno lauro pure di zucchero che l'ombrava; e interno era un ballo delle nove Muse, e Apollo con lira, pur di zucchero; e drieto a questo monte veniano venti poeti, dieci greci e dieci latini, a dua a dua, vestiti con suoi abiti ornatissimi; e ciascuno aveva uno libro in mano di buona grandezza, di zucchero, colle coperte colorite e serragli argentini e dorati che parevano veri libri: M. Tabarini (1870), Descrizione del convito e delle feste fatte in Pesaro per le nozze di Costanzo Sforza e di Camilla d'Aragona nel maggio 1475, Firenze, tip. di G. Barbèra, p. 52; C. Benporat (1999), Decori, castelli, op. cit., p. 44.

72 Prima comparse sopra d'uno asse lo Papa, el Principe, et lo Duca de Milano cum le arme loro, et quelle de la signoria vostra, poi Santo Marco deinde la Bissa et lo diamante et tante altre representatione de diverse cose tute lavorate de zucharo dorate che facevano el numero de 300 cum infiniti piatti de confectione, et cope da bevere in mese, li quali tuti se destenderono per la salla che fu uno bellissimo spectaculo: P. Molmenti (1880), La storia di Venezia nella vita privata dalle origini alla caduta della repubblica, Venezia, Roux e Favale, pp. 262, 609; A. Cougnet, op. cit., p. LXVII. 
costruzioni esistenti. Talvolta avevano molti elementi e particolari perciò sembravano assolutamente realistici. Ad esempio, nel maggio 1475, Costanzo Sforza, signore di Pesaro, commissionò simili castelli di zucchero con numerosi elementi imitanti le fortificazioni ${ }^{73}$. Un mese più tardi, nel giugno 1475 a Rimini, in occasione del matrimonio di Roberto Malatesta con Isabella di Montefeltro nell'ambito di una colazione dolce vennero preparati quaranta castelli di zucchero con sofisticati particolari imitanti fortificazioni. Un interesse particolare da parte dei convitati suscitò la miniatura di una fontana del luogo, che spargeva acqua profumata, nonché una replica in miniatura della porta di San Pietro o dell'arco di Ottaviano ${ }^{74}$.

Talvolta sui singoli elementi delle composizioni di zucchero venivano installati animali vivi: tale situazione ebbe luogo nel corso di un sontuoso banchetto di nozze in occasione del matrimonio di Annibale II Bentivoglio con Lucrezia d'Este, nel gennaio 1487, durante cui "fu portato un castello di zuccaro con li merli e torri artificiosamente composto pieno di uccelli vivi; il quale come fu posto nel mezzo della sala, uscirono fuore volando tutti gl'uccelli con gran piacere et diletto de' convitati" ${ }^{75}$. Non era l'unica composizione di zucchero. Dopo che vennero serviti vari piatti, descritti in seguito da Cherubino Ghirardacci (1519-1598):

vennero poi le torte di zuccaro con amandole, giuncate insieme con biscotti; addussero poi teste di capretti, tortore, pernici arrosto et poi un castello pieno di conigli, il quale, posato nella sala, uscirono fuore correndo chi qua chi là con risa e piacere de' convitati. Seguitarono poi dietro il castello pasteletti di conigli per cotal modo composti, che non parevano differenti puntini da quelli che dal detto castello erano usciti; poi portorono capponi pure vestiti. Poscia fu portato un artificioso castello ove era un grosso porco, et posto nel mezzo della sala, non potendo uscir fuori del castello, gridando drizzavasi in piedi, guardando per li merli hora uno et hora l'altro ruggendo, et così affaticandosi et gridando per fuggirsi, apparvero li scalchi con li servi con porchette cotte intiere dorate che in bocca tenevano un pomo, poi vennero arrosti di più sorti, anatre salvatiche et simili ${ }^{76}$.

73 C. Benporat (2001), Feste e banchetti..., pp. 176-223; C. Benporat (1993), "La convivialità rinascimentale alla corte degli Sforza", Appunti di gastronomia, 10, pp. 46-53.

74 P. Mantegazza (1871), Quadri della natura umana. Feste ed ebbrezze, vol. I, Milano, Presso Giuseppe Bernardoni Tipografo e la Libreria Brigola, p. 88.

75 C. Ghirardacci (1932), Historia di Bologna, [in:] Rerum Italicarum Scriptores, XXXIII, I, Città di Castello, Casa Editrice S. Lapi, p. 238.

76 C. Ghirardacci, op. cit., p. 238; C. Benporat (2001), Feste e banchetti..., p. 251; G. Conte don Gozzadini (1839), Memorie per la vita di Giovanni II Bentivoglio, Bologna, Belle Arti; A. Cougnet, op. cit., pp. LV, LXIV-LXV. 
Gli esempi di cui sopra indicano in modo chiaro che in territorio italiano la lavorazione dello zucchero raggiunse un livello avanzato. Sono anche una testimonianza delle capacità degli speziari del luogo, legati con le singole corti principesche. Si può costatare quindi che erano una prova concreta di una vera maestria di quel gruppo professionale. In tale prospettiva non c'è da meravigliarsi che i fenomeni descritti trovarono la loro espressione nella letteratura italiana dedicata alle questioni farmaceutiche (e soprattutto nelle famose farmacopee), che conobbe il suo sviluppo nella seconda metà del XV secolo.

Anche se in Italia nel XV secolo un ruolo fondamentale spettò all'opera di Saladino d'Ascoli Compendium aromatariorum, stampata nel 1488 e all'elenco ufficiale di medicinali pubblicato a Firenze nel 1498 (noto come Ricettario fiorentino), dobbiamo osservare che i temi riguardanti la produzione di dolci, che qui ci interessa in modo particolare, in ambedue le opere vennero trattate in modo assolutamente marginale ${ }^{77}$.

Nell'opera di un farmacista piemontese di Tortona, Quirico (e più propriamente Domenico) de Augustis, intitolata Lumen apothecariorum, la lavorazione dello zucchero viene trattata come un settore a parte dell'arte farmaceutica, o de facto dolciaria ${ }^{78}$. Anzi, sembra che per la prima volta a tale settore di attività e del sapere degli speziari italiani fu attribuito il nome artificium zucchari. Tale titolo proprio venne dato all'ultimo, quattordicesimo capitolo della menzionata farmacopea. In quel capitolo vennero raccolte 31 ricette di diversi tipi di confetti, frutta candita, confetture, marzapane, torroni e gelatine ${ }^{79}$.

Il carattere speciale della fonte consiste nel fatto che Domenico (Quirico) de Augustis per la prima volta nella letteratura farmaceutica raccolse le regole come dare il colore allo zucchero. Diede anche indicazioni indispensabili come plasmare pasta di zucchero per ottenere una forma e un aspetto concreto ${ }^{80}$.

77 Saladino Ferro d'Ascoli (2017), Compendium aromatariorum (ca. 1450). Alonso Rodríguez de Tudela, El compendio de los boticarios (1515). A Compendium for Apothecaries, [in:] Romania Mediterranea IV, Romance Philology, 71, Spring.

78 Q. de Augustis, op. cit.; Das Lumen apothecariorum von Quiricus de Augustis übersetzt und kritisch bearbeitet. Inaugural-Dissertation zur Erlangung des Doktorgrades der Naturwissenschaftlichen Fakultät der Ludwig-Maximilians-Universität München (1973), vorgelegt von Hugo Michael Wolf aus München, München, Dissertationsund Fotodruck Frank.

79 Q. de Augustis, op. cit., pp. XXXVv-XXXVIIr; Das Lumen, op. cit., pp. 281-293.

80 Q. de Augustis, op. cit., p. XXXVIr; traduzione italiana sta in: Lume de speciali, coposto dal sottilissimo dottore et medico, maestro Quirico de gli Augusti, di Tortona [in:] Luminare maggiore, utile et necessario a tutti li medici \& speciali, raccolto per Nicolo Mutoni medico, da molti eccellentissimi medici, con un breue commento di Iacobo Manlio, et il Lume, \& Tesoro de Speciali, In Vinegia 1559, fol. 53; Das Lumen, pp. 289-291. 
Oltre a ciò, da un'attenta lettura delle singole ricette risulta che l'autore non cercò solo di appoggiarsi sul sapere farmaceutico del suo tempo, relativo all'utilizzo dello zucchero, ma provò anche ad arricchire le singole ricette con le proprie e concrete osservazioni pratiche.

Ad esempio con tale situazione abbiamo a che fare nella ricetta delle caramelle di muschio:

I moscardini con difficultà pigliano forma, ma il sagace \& diligente speciale, nel misciare il zuccaro presto darà a queste specie. Ma quando gli hauerai dato due o tre mani di zuccaro bisogna criuerlarli, \& anderanno giù moscardini come ponte d'ago, \& poluere, lequali si conseruino. Gli altri c'hanno corpo si forniscano di cuoprire. Io ne feci fare un tratto da un fante bene esperto, \& ui stette presente, si che gli fece in breue tempo \& ottimi ma prima che gli fornisse lasciò la pelle delle mani al bacilone, \& quella che gli rimase era $\operatorname{secca}^{81}$.

In quel caso si può parlare del sapere e delle capacità particolari degli speziari italiani relativi all'appartenenza nazionale?

Per rispondere a questa domanda occorre fare prima unadeguata comparazione. Nel nostro caso il punto di riferimento saranno i territori della Corona d'Aragona. Proprio da quell'ambito culturale e linguistico proviene l'opera anonima Libre de totes maneres de confits, datata per la prima metà del $\mathrm{XV}$ secolo e che contiene 31 ricette di diversi prodotti dolciari ${ }^{82}$. Vi si trovarono uniche ricette per confetture, frutti canditi, marzapani, torroni. Senza entrare in particolari, si può constatare che, anche se la base per una parte di questi prodotti è lo zucchero, dobbiamo rilevare anche un importante ruolo del miele, in quanto ingrediente di base di alcune composizioni. Una di queste, rara però in Italia, composta da molti ingredienti era la confitura composta. Per la sua produzione si usavano alcune decine di ingredienti, e il prodotto finale

81 Q. de Augustis, op. cit., p. XXXVIr; Das Lumen, p. 285-286.

82 Llibre de totes maneres de confits (1946), [in:] Lluís Faraudo de Saint-Germain, „Llibre de totes maneres de confits. Un tratado manual cuatrocentista de arte de dulcería", Boletín de la Real Academia de Buenas Letras de Barcelona, vol. XIX; Llibre de totes maneres de confits (2003), [in:] Llibre de Sent Soví, Llibre de totes maneres de potatges de menjar, R. Grewe (a c. di), edició revisada per A. J. Soberanas i J. Santanach, Llibre de totes maneres de confits, edició critica de J. Santanach i Suñol, Barcelona, Barcino,; R. Hryszko (2010), „Llibre de totes maneres de confits - Księga o różnych sposobach przyrządzania słodyczy - starokataloński zbiór przepisów kulinarnych z XIV wieku”, Zeszyty Naukowe Uniwersytetu Jagiellońskiego, t. MCCCXII, Prace Historyczne, t. 137; R. Hryszko, R. Sasor, op. cit. 
consisteva nell'unione di semiprodotti dai gusti opposti, il che poteva far capire quali fossero le preferenze dell'élite della società medievale ${ }^{83}$.

Nella prospettiva delineata sopra, si vede in modo chiaro che la produzione degli speziari italiani, basata sullo zucchero, garantì un'ascesa particolare di quel settore, fino ai vertici di maestria. Ciò è dovuto al fatto che i rappresentanti di alcune nazioni italiane non solo avevano sotto controllo il commercio di questa materia, ma controllavano anche i principali centri di produzione. Laumento generale delle ricchezze e del benessere degli Stati italiani nel XIV e nel XV secolo, nonché i processi di trasformazioni politiche, economiche, sociali e culturali, influirono notevolmente sullallargamento del settore degli acquirenti dello zucchero e dei suoi prodotti. Le realizzazioni degli speziari italiani, basate sullo zucchero, considerate - conformemente alle linee della dietetica dell'epoca - come medicinali, cominciarono ad essere viste in una prospettiva completamente nuova. Il loro gusto e la loro forma erano a tal punto attraenti, che divennero un oggetto di desiderio da parte delle élite di quei tempi. In una vasta prospettiva delle trasformazioni culturali relative all'affermazione delle nuove correnti del pensiero o del costume, le modifiche del modo in cui viene inteso il posto e il ruolo dei prodotti dolciari può costituire un nuovo, anche se ancora poco conosciuto, contributo delle singole nazioni italiane allo sviluppo della cultura europea.

Traduzione: Bogumiła Bielańska

\section{Bibliografia}

André, Jacques, (1961). L'alimentation et la cuisine à Rome, Paris, C. Klincksieck.

Ausécache, Mireille (2007). "Manuscrits d’antidotaires médiévaux: quelques exemples du fonds latin de la Bibliothèque nationale de France”, Médiévales, 52, printemps, pp. 55-74.

Balletto, Laura (1986). Medici e farmaci, scongiuri ed incantesimi, dieta e gastronomia nel medioevo genovese, Genova, Università di Genova.

Beccadelli, Antonio, el Panoramita (1990). Dels fets e dits del gran rey Alfonso, Barcelona, Editorial Barcino.

Bénézet, Jean-Paul (1999). Pharmacie et médicament en Méditéranée occidentale (XIII-XVI siècles), Paris, Honoré Champion.

Benporat, Claudio (1993). "La convivialità rinascimentale alla corte degli Sforza", Appunti di gastronomia, 10, pp. 46-53.

Benporat, Claudio (1999). "Convivialità. cucina e decori delle tavole nella Firenze del'400", Appunti di gastronomia, 28, pp. 5-21.

Benporat, Claudio (1999). "Decori, castelli e statue di zuccharo sulla scena conviviale italiana", Appunti di gastronomia, 30, pp. 42-67.

83 Llibre (1946), op. cit., pp. 113-114; Llibre (2003), op. cit., p. 84; R. Hryszko (2010), op. cit., pp. 197-198; R. Hryszko, R. Sasor, op. cit., pp. 141-143. 
Benporat, Claudio (2001). Feste e banchetti. Convivialità italiana fra Tre e Quattrocento, Firenze, Leo S. Olschki Editore.

Benporat, Claudio (2001). "De conviventia, un trattato di etica conviviale di Giovanni Pontano", Appunti di gastronomia, 36, pp. 10-26.

Benporat, Claudio (2005). "Note per la storia dei confetti", Appunti di gastronomia, 47, pp. 71-89.

Benporat, Claudio (2007). "La „collatione". una nuova forma conviviale”, Appunti di gastronomia, 52 , pp. $45-58$.

Benporat, Claudio (2009). "Convivialità degli ultimi Visconti", Appunti di gastronomia, 58, pp. 5-16.

Carnevale Schianca, Enrico (2011). La cucina medievale. Lessico, storia, preparazioni, Firenze, Leo S. Olschki Editore.

Ciasca, Raffaele (1927/1977). L'arte dei medici e speziali nella storia e nel commercio fiorentino dal secolo XII al XV, Firenze, Leo S. Olschki Editore.

Cifuentes i Comamala Lluís (2006). La ciència en català a l'edat mitjana i el renaixement, Barcelona, Publicacions i edicions de la Universitat de Barcelona.

Claramunt, Salvador (1988). "Dos aspectes de l'alimentació medieval: dels canonges a les „miserabiles personae”, [in:] Alimentació i societat a la Catalunya medieval. Anuario de estudios medievales, Anex 20, Barcelona, Consejo Superior de Investigaciones Sientíficas, pp. 167-172.

Comolli Carlo (1991). "Cucina e feste presso la corte ferrarese del'400”, Appunti di gastronomia, 6, pp. 23-30.

Cougnet, Alberto (1923). L'evoluzione dell'arte dulciaria in Italia, [in:] Giuseppe Ciocca, Il pasticciere e confettiere moderno. Raccolta completa di ricette, Milano, Ulrico Hoepli, pp. XIX-LX.

Das Lumen apothecariorum von Quiricus de Augustis übersetzt und kritisch bearbeitet. InauguralDissertation zur Erlangung des Doktorgrades der Naturwissenschaftlichen Fakultät der Ludwig-Maximilians-Universität München (1973). Vorgelegt von Hugo Michael Wolf aus München, München, Dissertations- und Fotodruck Frank.

Dorveaux, Paul (1896). L'Antidotaire Nicolas. Deux traductions françaises de l'Antidotarium Nicolai, l'une de XIV siècle, suivie de quelques recettes de la même époque et d'un glossaire, l'autre de $X V^{\mathrm{e}}$ siècle, incomplète, Paris, $\mathrm{H}$. Welter.

Faccio, Bartolomeo (1590). I fatti d'Alfonso d'Aragona primo re di Napoli, Vinegia, Apresso Giovanni et Gio. Paolo Giolitti de Ferrari.

Faccioli, Emilio (1966). Arte della cucina, vol. I, Milano, Edizioni il Polifilio.

Gajda, Zbigniew (2011). Do historii medycyny wprowadzenie, Kraków, Wydawnictwo WAM.

Gandini, Luigi Alberto (1899). Tavola, cucina e cantina della corte di Ferrara nel Quattrocento. Saggio storico, Modena, Società tipografica modenese.

Ghirardacci, Cherubino (1932). Historia di Bologna, [in:] Rerum Italicarum Scriptores, XXXIII, I, Città di Castello, Casa Editrice S. Lapi.

Giuffrida, Antonino (1976). La bottega dello speziale nelle città siciliane del'400, [in:] Atti del Coloquio Internazionale di Archeologia Medievale, Palermo-Erice, 20-22 settembre 1974, Palermo, Istituto di storia medievale, Unversità di Palermo, pp. 465-504.

Gozzadini, Giovanni, Conte don (1839). Memorie per la vita di Giovanni II Bentivoglio, Bologna, Belle Arti. 
Guiral, Jacqueline (1984). "Le sucre à Valence aux XV et XVI siècles", [in:] Manger et boire au Moyen Âge. Actes du Collque de Nice (15-17 octobre 1982), t. 1: Aliements et Societé, Nice, Les Belles Lettres.

Hryszko, Rafał (2010). „Llibre de totes maneres de confits - Księga o różnych sposobach przyrządzania słodyczy - starokataloński zbiór przepisów kulinarnych z XIV wieku”, Zeszyty Naukowe Uniwersytetu Jagiellońskiego, t. MCCCXII, Prace Historyczne, t. 137, pp. 176-206.

Hryszko, Rafał (2013). Media aeva dulcia. Analiza produkcji i konsumpcji w Koronie Aragonii w XIV i XV w., Kraków, Towarzystwo Wydawnicze "Historia Iagellonica".

Hryszko, Rafał (2012). Na kaffeńskim stole-wyimek z problematyki żywieniowej czarnomorskich kolonii Genui w późnym średniowieczu, [in:] Łukasz Gędłek, Tomasz Krzyżowski, Michał Michalski (a c. di), Regiones Euxinum Spectantes. Stosunki kulturowe, etniczne i religijne na przestrzeni dziejów, Kraków, Uniwersytet Papieski Jana Pawła II, pp. 237-255.

Hryszko, Rafał (2019). „The Sweet War, or How Alfons V of Aragon's Military Campaigns Affected the Eating Habits in Early to Mid-15th Century", Perspektywy kultury, nr 26 (3/2019), pp. 133-157.

Hryszko, Rafał e Sasor, Rozalia (2017). Średniowieczne słodycze katalońskie w źródtach i literaturze ( $z$ wyborem tekstów z XIV i XV wieku), Kraków, Towarzystwo Wydawnicze Historia Iagellonica.

Hyman, Mary (1992). "Les «menues choses qui ne sont de nécessité»: les confitures et la table", [in:] Du manuscrit à la table, essais sur la cuisine au Moyen Age et répertoire des manuscrits médiévaux contenant des recettes culinaires, sous la direction de C. Lambert, Montréal, Champion-Slatkine, Les Presses de l’Université de Montréal, pp. 273-283.

Jacquart, Danielle (2005). „Wpływ medycyny arabskiej na średniowieczny Zachód”, [in:] Historia nauki arabskiej, Roshdi Rashed (a c. di), t. 3: Technika, alchemia, nauki przyrodnicze i medycyna, Warszawa, Wydawnictwo Akademickie Dialog, pp. 222-238.

Kronika medycyny (1994). Warszawa, Kronika.

Kuhne, Rosa (1994). "El azúcar: usos dietéticos y farmacéuticos según los médicos árabes medievales", [in:] 1492: lo dulce en la conquista de Europa. Actas del quarto seminario internacional sobre la caña de azúcar, Motril 21-25 septembre 1992, Granada, Diputación General de Granada, pp. 41-62.

Kuhne Brabant, Rosa (1997). “Le sucre et le doux dans l'alimentation d'al-Andalus”, Médiévales, 33, automne, pp. 55-67.

Leone, Simona (2009). "Alcuni appunti sul torrone”, Appunti di gastronomia, 58, pp. 83-92.

Llibre de totes maneres de confits (1946). [in:] Lluís Faraudo de Saint-Germain, „Llibre de totes maneres de confits. Un tratado manual cuatrocentista de arte de dulcería”, Boletín de la Real Academia de Buenas Letras de Barcelona, vol. XIX, pp. 105-121.

Llibre de totes maneres de confits (2003). [in:] Llibre de Sent Soví, Llibre de totes maneres de potatges de menjar, a cura de R. Grewe, edició revisada per A. J. Soberanas i J. Santanach, Llibre de totes maneres de confits, edició critica de J. Santanach i Suñol, Barcelona, Barcino, pp. 273-295.

Lume de speciali (1559). Lume de speciali, composto dal sottilissimo dottore et medico, maestro Quirico de gli Augusti, di Tortona [in:] Luminare maggiore, utile et necessario a tutti li medici \& speciali, raccolto per Nicolo Mutoni medico, da molti eccelentissimi medici, con un breue commento di Iacobo Manlio, et il Lume, \& Tesoro de Speciali, In Vinegia, Giovanni Bariletto. 
Maestro Martino (1996). Ms. Bühler 19, New York, Pierpont Mogran Library, [in:] Claudio Benporat, Cucina italiana del Quatrocento, Firenze, Leo S. Olschki Editore, pp. 233-292.

Maggi, Bruno (2001). "Il marzapane", Appunti di gastronomia, nr 34, 2001, s. 73-84.

Mancusi Sorrentino, Lejla (1993). "Apparecchi diversi da mangiare et rimedii”, Appunti di gastronomia, 11, pp. 18-104.

Mantegazza, Paolo (1871). Quadri della natura umana. Feste ed ebbrezze, vol. I, Milano, Presso Giuseppe Bernardoni Tipografo e la Libreria Brigola.

Mensajeros barceloneses en la corte de Nápoles de Alfonso V de Aragón (1963). Introducción y texto por J.M. Madurell Marimón, Barcelona, Consejo Superior de Investigaciones Sientíficas.

Molmenti Pompeo (1880). La storia di Venezia nella vita privata dalle origini alla caduta della repubblica, Venezia, Roux e Favale.

Notabilia temporum di Angelo de Tummulillis da Sant'Elia (1890). C. Corvisieri (a c. di), Roma, Tipografia Francesco Vigo.

Ouerfelli, Mohamed (2008). Le sucre. Production, commercialisation et usage dans Méditerranée médiévale, Leiden-Boston, Brill.

Palmero, Giuseppe (1998). Entre culture thérapeutique et culture matérielle: les domaines de savoir d'un anonyme gênois à la fin du Moyen-Âge. Le manuscrit inédit „Medicinalia quam plurima”, vol. II, thèse de doctorat sous la direction de Henri Bresc.

Plouvier, Lilianne (1988). "La confiserie européenne au Moyen Âge", Médium Aevum quotidianum, 13, pp. 28-47.

Plouvier, Lilianne (1992). "Le «letuaire», une confiture du bas Moyen Âge”, [in:] Du manuscrit à la table, essais sur la cuisine au Moyen Age et répertoire des manuscrits médiévaux contenant des recettes culinaires, sous la direction de C. Lambert, Montréal, Champion-Slatkine, Le Presses de l'Université de Montréal, pp. 243-256.

Plouvier, Lilianne (1999). "L'introduction du sucre en pharmacie", Revue d'histoire de la pharmacie, 87 (332), pp. 199-216.

Plouvier, Lilianne (2006). "Le rôle d'al-Andalus dans la transmission des connaissances de l'Orient vers l'Occident: l'exemple e la confiserie”, Horizons maghrébins, 55, pp. 30-47.

Pontano, Giovanni Gioviano [Ioannis Ioviani Pontani] (1518). Opera omnia soluta oratione composita, t. I, Venetiis.

Quiricus de Augustis (1492). Lumen apothecariorum, Taurini.

Regimen sanitatis Magini mediolanensis cum nonnulis insuper Auicenne ac plerumq[ue] aliorum auctoru $[\mathrm{m}]$ margine cartharum insertis; Insuper opusculum de fleubothomia editu[m] a Reginaldo de Villa noua, (1506). per Gaspardum Philips, expensis Iohannis Petit, Parisium.

Saladino Ferro d'Ascoli (2017). Compendium aromatariorum (ca. 1450). Alonso Rodríguez de Tudela, El compendio de los boticarios (1515). A Compendium for Apothecaries, [in:] Romania Mediterranea IV, Romance Philology, 71, Spring.

Santoro, Daniela (2006). "Lo speciale siciliano tra continuità e innovazione: capitoli e costituzioni dal XIV al XVI secolo”, Mediterranea. Ricerche storiche, 3, dicembre, pp. 465-484.

Savage-Smith, Emilie (2005). Medycyna, [in:] Historia nauki arabskiej, Roshdi Rashed (a c. di), t. 3: Technika, alchemia, nauki przyrodnicze i medycyna, Warszawa, Wydawnictwo Akademickie Dialog, pp. 161-221. 
Silberman, Henri C. (1994). "Un électuaire d'Avicenne ou de la difficulté d'identifier les constituants de médicaments antiques”, Revue d'histoire de la pharmacie, 82 (301), pp. $132-147$.

Sorrentino, Francesco (2001). "Le nozze Trivulzio-d'Avalos e la pignolata napoletana", Appunti di gastronomia, 44, pp. 57-62.

Tabarini, Marco (1870). Descrizione del convito e delle feste fatte in Pesaro per le nozze di Costanzo Sforza e di Camilla d'Aragona nel maggio 1475, Firenze, tip. di G. Barbèra.

Vela i Aulesa, Carles (1996). "La col-lació, un apat medieval poc conegut", [in:] La Mediterrània. àrea de convergència de sistemes alimentaris (segles V-XVIII). XIV Jornades d'Estudis Històrics Locals: realizades a Palma del 29 de novembre al 2 dicembre de 1995), Palma, Consejo Superior de Investigaciones Sientíficas, pp. 669-686.

Vigna, Amadeo (1879). Codice diplomatico delle colonie tauro-liguri durante la signoria dell'Ufficio di San Giorgio (MCCCCLIII-MCCCCLXXV), t. II, p. II (anni 1473-1475). Atti della Società Ligure di Storia Patria, vol. VII, p. II, fasc. I-II.

\section{Sitografia}

http://dbe.rah.es/biografias/6367/alfonso-v [10/11/2019].

https://encyklopedia.pwn.pl/haslo/3888384/cukiernictwo.html [04/12/2019].

https://www.treccani.it/enciclopedia/alfonso-v-d-aragona-re-di-sicilia-re-di-napoli_

(Dizionario-Biografico) [10/11/2019].

http://encyklopedia.pwn.pl/haslo/3888384/cukiernictwo.html https://www.treccani.it/

enciclopedia/maino-maineri_(dizionario_biografico) [10/11/2019].

https://www.treccani.it/vocabolario/pasticceria [04/12/2019].

https://www.treccani.it/vocabolario/confetteria [04/12/2019].

https://www.treccani.it/vocabolario/confetto2 [04/12/2019].

https://www.treccani.it/vocabolario/spezie [04/12/2019].

https://www.treccani.it/vocabolario/speziale [04/12/2019].

https://www.treccani.it/vocabolario/speziale2 [04/12/2019]. 